\title{
Research on the Domestic and Foreign Progress and Trend of Cyberchondria
}

\author{
Wu Haibin ${ }^{1}$, Fan Mingyue ${ }^{1, *}$ \\ ${ }^{1}$ Jiangsu University, School of Management, San Jiang Building 1502, China
}

\begin{abstract}
Cyberchondria is an emerging risk in the Internet age. It causes a serious threat to public health and the social environment. At present, the domestic and foreign related studies are in the initial stage, so few of systematic review and summary are shown. Based on the core collection database of China National Knowledge Infrastructure (CNKI) and Web of Science (WOS) as the data source, this paper uses CiteSpace to explore the domestic and foreign research developments and hotspots in the field of cyberchondria. By comparing and summarizing the domestic and overseas research status and hot spots of cyberchondria, this paper puts forward the research direction of cyberchondria in the future, in order to promote the further development of related research.
\end{abstract}

\section{Introduction}

The 46th Statistical Report on the Development Status of China's Internet [1] pointed out that as of June 2020, the scale of online medical users in China reached 276 million, accounting for $29.4 \%$ of the total number of Internet users. With the increase in the number of Internet users, the problem of online health information search has come with the gradual increase in the number of patients with cyberchondria. Especially during the COVID-19 pandemic, a large amount of unconfirmed news was scattered on the Internet, which intensified people's concern about their health problems. Anxiety, paranoid, and the resulting strained doctor-patient relationship have posed a serious threat to public health and the social environment. Therefore, as an "emerging risk" in the Internet era, it is necessary to review and summarize the current state of research domestic and abroad, which is important to further promote research and introduce effective interventions and treatment measures for cyberchondria in the future.

\section{Concepts related to cyberchondria}

The concept of cyberchondria was first mentioned in 2001 in the article "A new disorder, cyberchondria, sweeps the Internet" published in the British newspaper The Independent. The term "cyberchondria" is a combination of the words "cyber" and "hypochondriasis". As an emerging disease in the Internet era, it has gradually attracted the attention of academics. Various definitions of this phenomenon have been proposed by domestic and international scholars [2], but the more accepted concept nowadays is that cyberchondria is an excessive or repetitive search for health-related information online out of health distress and anxiety, which results in amplifying the symptoms of such distress or anxiety.

At present, domestic and foreign research on cyberchondria is still in its infancy, and on the basis of previous research, scholars domestic and abroad have used qualitative analysis to review research related to cyberchondria, however, in the face of the huge amount of literature, the analysis conducted by reading, summarizing and concluding has certain subjectivity, one-sidedness and limitations. Therefore, as the research related to cyberchondria has developed so far, what are the overall research trends domestic and abroad? What are the research hotspots? What is the direction of future research? Addressing these questions will help promote the in-depth research and exploration of cyberchondria by scholars domestic and abroad.

In recent years, CiteSpace visualization software can objectively and scientifically present the research history and development trends of an academic field through knowledge mapping technology, avoiding the bias caused by bibliometric analysis methods on the assessment of literature value in quantitative analysis [3]. Based on this, this paper uses this software to analyze and summarize the current situation and frontier hotspots of research on cyberchondria domestic and abroad, in order to deepen the understanding and grasp of the research progress of cyberchondria and provide reference for the subsequent research work.

\section{Data sources and research methods}

\subsection{Data sources}

In this paper, the core collection database of China National Knowledge Infrastructure (CNKI) and Web of

\footnotetext{
* Corresponding author: fanmy@ujs.edu.cn
} 
Science (WoS) was used as the search platform, and the search date was "September 12, 2020". In CNKI, the search was conducted with the subject terms of "cyberchondria", "internet hypochondria", and a total of 10 valid articles were retrieved. In WoS, the search terms were "cyberchondria", "cyberchondriasis", "internet hypochondria", "online hypochondria", the literature categories were set to Article and Review, and a total of 65 valid articles were retrieved.

\subsection{Research methods}

This paper uses bibliometric analysis as the main research method. First, the annual distribution of domestic and international literature publications was statistically examined. Second, CiteSpace software was used to analyze the overview of the development of domestic and international research on cyberchondria. Third, analyze the hot topics of domestic and international research on cyberchondria. Finally, summarize the frontier hot spots and future research trends of cyberchondria.

\section{Overview of the development of domestic and international research on cyberchondria}

The analysis of the trend of the number of cyberchondria-related literature in CNKI and WoS from 2003 to 2020 (Figure 1) shows that the number of articles in the field of cyberchondria research domestic and abroad has basically maintained an increasing trend, and there are three obvious time periods that deserve attention. (1) From 2003 to 2011, the number of domestic and foreign literature on cyberchondria was relatively small (4 foreign articles and 1 domestic article), indicating that cyberchondria had not yet received academic attention during this time period. The main reason may be due to the fact that the Internet was not yet widespread at that time, the willingness of the public to search for online health information was low, and the phenomenon of cyberchondria was low. (2) During 2012-2018, the number of foreign publications increased significantly ( 30 articles), and the number of domestic publications also increased, but with a smaller increase (4 articles), which indicates that cyberchondria has received attention and recognition from foreign academics during this time period, and relevant research gradually emerged. (3) So far in 2019, the number of foreign literature on research related to cyberchondria has increased rapidly (31 articles), and the number of domestic publications has also increased significantly (5 articles), indicating that academics have become more active in research on cyberchondria and have continued to expand in depth. This is due to the increasing attention to the issue of cyberchondria as a result of the rapid popularity of the Internet and people's continued concern about their health, leading to increasing online health information-seeking behaviors.

At the national level, the United States was the most published country with 20 articles, along with other high-volume countries such as the United Kingdom, China, Australia, and Germany, constitute the main countries in cyberchondria research, but the countries are more loosely connected and have less collaborative research. Baylor University, Sydney University, and Florida State University are the major research institutions for cyberchondria, and these institutions account for a large proportion of the literature in the field, and the relevant literature is mainly published in medical, psychological, and behavioral journals, laying the foundation of the discipline in this field. Foreign scholars Fergus T A, Starcvic V and White R W are the core authors of the research in this field, and their published literature has made a high academic contribution to the study of cyberchondria.

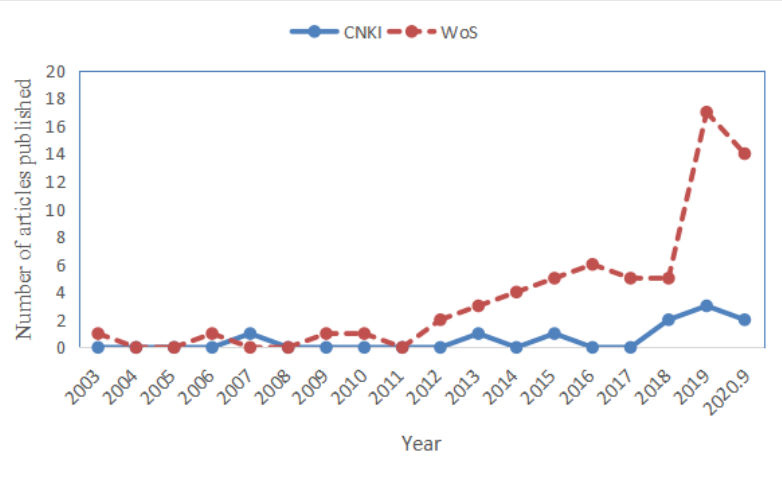

Fig. 1. Change in the number of cyberchondria literature in CNKI and WoS from 2003 to 2020

\section{Analysis of domestic and international research hotspots on cyberchondria}

\subsection{Keyword co-occurrence analysis}

In scientometrics, the research hotspots in academic fields are usually determined by the frequency of keyword occurrences [4]. The top three keywords that appear frequently in domestic cyberchondria are cyberchondria, health anxiety and hypochondria, which appear 5 times, 4 times and 3 times respectively, and they represent the hotspots of domestic research. The subjects of domestic research on cyberchondria are mainly college students; the research content is focused on the fields of psychology and medicine, and supplemented by analysis of online information-seeking and other behaviors.

The keyword contribution mapping of foreign cyberchondria is shown in Fig 2, in which cyberchondria, health anxiety and internet appear most frequently, which is basically consistent with the high-frequency keywords of domestic studies. The main subjects of foreign studies include: adolescent, young people and medical patient; research methods include: experimentation and questionnaire; research contents include: internet, information, health anxiety, information seeking, hypochondriasis and potential risk factor. It can be seen that due to the high volume of foreign scholars' publications in this field, their research 
perspectives and research contents have shown a diversity beyond the fields of psychology and medicine, gradually transitioning to research fields oriented to information and Internet characteristics.

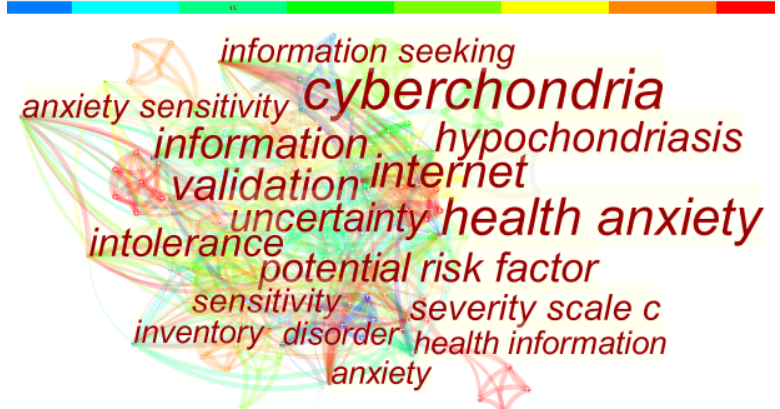

Fig. 2. Foreign cyberchondria keyword co-occurrence mapping

\subsection{Hot topic analysis}

Due to the low volume of postings, only one cluster \#0 health anxiety was generated for the keywords of cyberchondria in China. The representative keywords of this cluster are cyberchondria, online health information, health anxiety and online health information search, etc. The main focus is on the concept of cyberchondria, its influencing factors, measurement methods and its relationship with health anxiety. Based on the definition of the concept of cyberchondria, some scholars found that health anxiety, intolerable uncertainty, perfectionist traits, perceived information credibility, cognitive bias, and online search technology are the main influencing factors of cyberchondria, and considered cyberchondria as an extension of health anxiety in the Internet era [5].

The keyword clustering mapping of foreign cyberchondria is shown in Fig 3. Combining the clustering results and the relevant literature, it can be seen that the research content related to foreign cyberchondria can be divided into two major hot topics.

Hot Topic 1: The mechanism, symptom manifestations, and interventions of cyberchondria. This research theme contains clusters \#2illness anxiety, \#3online health information seeking, \#6cyberchondriasis, and clusters \#5 and \#9 with the same googling result. The representative keywords are health information, internet searching, health anxiety, worry, and hypochondriasis. To date, the Internet has become the most popular place for health information sources. The low cost, convenience, and anonymity of online health information seeking have attracted an increasing number of users to use the Internet to obtain health-related information[6]. However, from the social level, due to the limited supervision and management of online information by the government and other departments, the quality of online health information is somewhat problematic, and many unconfirmed information is widely disseminated in the Internet, which may lead to users' misconceptions about health and anxiety about disease concerns [7], thus making cyberchondria more and more prevalent. At the individual level, users with certain cognitive biases in disease beliefs are prone to health anxiety, and this anxiety may further exacerbate search behavior [8], ultimately leading to the development of cyberchondria. Possible symptoms of cyberchondria are manifested in anxiety, internet addiction, dysfunction, and low quality of life [9], and these issues should be of concern to society and individuals. Prevention and intervention measures for cyberchondria have been proposed by foreign scholars, such as improving users' health information literacy through education to reduce the frequency of searching for online health information; strict auditing and supervision of internet-related health information by relevant departments and institutions to reduce the possibility of negative results from incorrectly searching for health information; and developing more health apps to provide users with accurate and reliable and by developing more health apps to provide users with accurate and reliable information to reduce unnecessary concerns about online health information [2]. However, the relevant measures lack operability and effectiveness of the exploration. Therefore, on the basis of clarifying the theories and mechanisms related to cyberchondria, future research can establish effective paths for prevention and intervention of cyberchondria at different levels, such as individuals, governments and enterprises.

Hot Topic 2: The various factors that influence cyberchondria and the relationship between them. This research topic includes the clusters \#0problematic internet use, \#1 information exposure, \#4anxiety sensitivity, \#7defensive pessimism, and \#8misinformation. The representative keywords are open data, anxiety, negative affectivity and information overload. Cyberchondria can be greatly influenced by external circumstances, and in the context of the COVID-19 outbreak, a large amount of fake news and misinformation has been revealed on the Internet, affecting people's judgments about their health and increasing their concerns. Several studies have pointed out that there is a positive association between problematic internet use, people's level of trust in online information, information overload, obsessivecompulsive disorder, anxiety sensitivity, intolerable uncertainty, defensive pessimism, neuroticism and low self-esteem[5,10,11,12,13], all of which positively influence the development and progression of cyberchondria. As can be seen, foreign research on the influencing factors of cyberchondria is growing centrally, but the comprehensiveness and universality of the influencing factors need to be further explored because there is still no universally agreed measurement scale and measurement method. In addition, existing studies have also neglected the interactions among the influencing factors and the theoretical basis of their effects [12], which has limitations for the systematic study of cyberchondria. Therefore, in future research, the influencing factors of cyberchondria as well as the influencing mechanisms can be clarified from different disciplinary perspectives. 


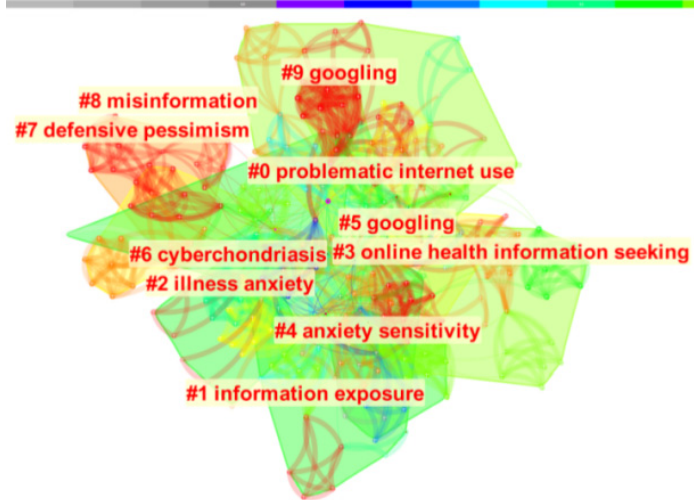

Fig. 3. Clustering mapping of foreign cyberchondria keywords.

\section{Conclusions}

The rapid development of the Internet era has led to the emergence of social problems based on the Internet, and cyberchondria is an emerging research area in this context. In this paper, with the help of CiteSpace software, we conducted a visual econometric analysis of the literature related to cyberchondria included in CNKI and WoS during 2003-2020, and the following conclusions were obtained.

(1) In recent years, scholars domestic and abroad have been paying more and more attention to the study of cyberchondria. Although there is a large gap between the number of papers published in China and abroad related to cyberchondria, the overall number of papers published is on the rise and is still increasing.

(2) Foreign scholars have shown great enthusiasm for research on cyberchondria, mainly in Western countries such as the United States, the United Kingdom, and Australia, with more active institutions such as Baylor University, Sydney University, and Florida State University, and related articles are mainly published in medical, psychological, and psychiatric journals, laying the disciplinary foundation for cyberchondria.

(3) Highly productive and influential literature mainly comes from some foreign experts and scholars and their research teams, and their research results play an important role in advancing research in the field of cyberchondria. The research on cyberchondria in China started late and has not yet formed a systematic and continuous research, so there is a big gap between the research results and the influential contributions from western countries.

(4) From the distribution of high-frequency keywords and hotspots, most of the hotspots of research on cyberchondria by scholars domestic and abroad in recent years are in the areas of concept definition, factor analysis, behavior analysis and measurement analysis. At the same time, it also reflects some integration and crossover with other disciplines, such as exploring the problems of doctor-patient relationship due to cyberchondria from the perspective of sociology, and exploring the dissemination of Internet health information from the perspective of communication, which indicates that the research system of cyberchondria has gradually matured.
(5) By clustering keywords, we found that the mechanism of cyberchondria, influencing factors and interventions remain the key research directions for scholars in the future, but at the same time, we need to further clarify the causal relationship between cyberchondria and its influencing factors, improve and unify the measurement tools and theoretical basis of cyberchondria, and verify the effectiveness of prevention and interventions in order to promote a comprehensive The study was conducted to further clarify the causal relationship between cyberchondria and its influencing factors.

With the rapid development of information technology and Internet technology, it is of great significance for public health and social environment to fully understand the generation mechanism, influencing factors and interventions of cyberchondria. At present, cyberchondria is still an emerging research field, and the research of domestic and foreign scholars is still in its initial stage, especially in the clarification of the theoretical system of cyberchondria, the influence mechanism, the development and validation of measurement tools, and the multidisciplinary integration analysis, etc. There is still much room for development and further research and practice.

\section{References}

1. China Internet Network Information Center, "The 46th Statistical Report on Internet Development in China," [EB/OL].[2020-09-29]. http://www.gov.cn/xinwen/2020/09/29/content_554 8176.htm. (In Chinese)

2. V. Starcevic, D. Berle. Expert Rev Neurother, 13, 205-213 (2013)

3. L. C. Freeman. Soc Networks, 1, 215-239 (1979)

4. C. Chen. J Anxiety Disord, 31, 58-64 (2015)

5. J. L. Ding, Z. H. Yang. Chinese Med, 53, 1984-1988 (2015)

6. V. Starcevic, S. Baggio, D. Berle, Y. Khazaal, K. Viswasam. Psychiat Quart, 90, 491-505 (2019)

7. X. L. Luo, J. T. Han, W. G. Fan, X. Li. Inf Doc Serv, 40, 76-86 (2019) (In Chinese)

8. R. D. McMullan, D. Berle, S. Arnáez, V. Starcevic. J Affect Disorders, 245, 270-278 (2019)

9. B. M. Mathes, A. M. N. P. Norr, Allan, B. J. Albanese, N. B. Schmidt. Psychiat Res, 261, 204-211 (2018)

10. T. A. Fergus. J Anxiety Disord, 28, 504-510 (2014)

11. T. A. Fergus, M. M. Spada. Clin Psychol Psychot, 24, 1322-1330 (2017)

12. S. Laato, A. K. M. N. Islam, M. N. Islam, E. EUR J Inform Syst, 29, 288-30 (2020)

13. T. A. Fergus. Cyberpsych Beh Soc N, 16, 735-739 (2013) 